\title{
Sonolência diurna excessiva em pré-vestibulandos
}

Excessive daytime sleepiness in senior high school students

José Carlos Souza', Neomar Souza', Ester Shiori Hirai Arashiro', Rafaela Schaedler ${ }^{1}$

\section{RESUMO}

Objetivo: O sono é um fenômeno que interfere nos aspectos cognitivos. O objetivo deste estudo foi avaliar a prevalência da sonolência diurna excessiva (SDE) em pré-vestibulandos de Campo Grande, MS. Métodos: Foram entrevistados 378 alunos com a escala de sonolência Epworth (ESE). As variáveis foram: sexo, uso esporádico de bebidas alcoólicas e fumo, relato de sinais e sintomas depressivos, renda familiar total dos membros do lar e idade. Foram empregados os testes qui-quadrado e de análise de variância. Resultados: Em relação ao gênero, 50,3\% eram homens e 49,7\% mulheres; 39,2\% ingeriam álcool; 6,6\% fumavam e 33\% já tinham tido depressão na vida. Entre os alunos, 55,8\% tinham SDE, 5,3\% eram indicativos de ter distúrbio respiratório ou síndrome da apnéia do sono. Foram detectadas associações entre as variáveis consumo de álcool e tabagismo, em relação à ESE.

\section{Palavras-chave}

Sonolência diurna excessiva, depressão, estudantes.

\section{Key-words}

Excessive daytime sleepiness, depression, students.
Conclusões: Foi alta a prevalência de SDE, sendo detectadas associações entre as variáveis uso esporádico de álcool e fumo, em relação à ESE. Novos estudos devem ser realizados a fim de prevenir as alterações cognitivas entre os pré-vestibulandos que apresentam SDE ou outro distúrbio do sono.

\section{ABSTRACT}

Objective: Sleep is a phenomenon that has influence on cognitive aspects. The purpose of the present study was to evaluate the excessive daytime sleepiness (EDS) prevalence. Methods: 378 senior high school students from a school of Campo Grande-MS, Brazil, were interview with the Epworth Sleepiness Scale. The variables that were crosSDE with the scale were: sex, alcohol use, smoking, symptoms of depression, family income and age. For the analysis it was USDE the $x^{2}$ test and the variance analysis test. Results: Our sample consisted of 50,3\% boys and 49,7\% girls, $39,2 \%$ were alcohol drinkers, 6,6\% were smokers and $33 \%$ had already had depression. The overall prevalence of EDS was 55,8\% and of respiratory disturbance or sleep apnea was 5,3\%. We found relationships between alcohol intake and smoking and the Epworth scale. Conclusions: It was high the prevalence of EDS, and associations between the variables sporadic consume of alcohol and tobaco, were detected, related to the ESE. New studies are needed to prevent cognitive changes among senior high school students that show ESS or another sleep disturbance.

1 Universidade Católica Dom Bosco (UCDB). 


\section{INTRODUÇÃO}

Durante o processo do dormir ocorrem modificações fisiológicas e comportamentais. Há, também, interferência direta nos processos cognitivos e de aprendizagem (Souza, 1999). Em relação aos distúrbios do sono, entre os mais freqüentes está a sonolência diurna excessiva (SDE), que afeta de 4\% a 12\% da população geral (Benbadis, 1998; Guilleminaulte e Stoohs; Clerk, 1993; Souza et al., 2002). Pessoas com SDE apresentam estresse psicológico, diminuição da produtividade no trabaIho ou na escola e aumento do risco de acidentes (Akerstedt et al., 2001; Canani e Barreto, 2001; Connor et al., 2001; Drummer et al., 2003; Ferreira, 2003; Häkkänen et al., 1999; McCartt et al., 2000); têm ainda prejuízos no funcionamento físico, na vitalidade e na qualidade de vida (QV) em geral (Beusterien et al., 1999; Franco et al., 2000; Glina et al., 2001; Häkkänen e Summala, 2000; Krieger, 2000; McArdle et al., 2001; Melamed e Oksenberg, 2002; Saletu et al., 2001; Yang et al., 2000).

Bulgari e Freitas (2001) relatam que os adolescentes são o grupo que apresentam maior privação de sono, apresentando, portanto, uma tendência a sonolência diurna. Mostram que os horários escolares e dos programas de fim de semana atendem geralmente às necessidades da família e não às necessidades dos adolescentes. Os autores destacam que a mudança de hábitos na hora de dormir durante a semana e também nos fins de semana são fatores estimulantes não só para a privação do sono, como também para a sonolência diurna.

Objetivou-se, neste estudo, avaliar a prevalência e os fatores predisponentes da SDE em pré-vestibulandos.

\section{MÉTODO}

A pesquisa foi realizada em uma instituição particular de ensino, em uma população de 378 alunos de todas as turmas do 3 o ano do ensino médio, por meio de amostragem por conveniência, no período de uma semana, na segunda quinzena do mês de maio de 2005. Não participaram da pesquisa apenas os alunos ausentes naqueles dias de aula.

Foi realizado um estudo descritivo e de corte transversal, mediante o levantamento de dados sociodemográficos (idade, sexo, renda familiar total dos membros do lar, uso esporádico de bebidas alcoólicas e fumo e relato de sinais depressivos) e da aplicação da escala de sonolência de Epworth (ESE). Entre as mais empregadas, mundialmente, está a ESE, desenvolvida por Murray W. Johns, no Centro de Distúrbios do Sono do Epworth Hospital, Melbourne, Austrália (Johns, 1991). Esta foi planejada como um questionário simples, de fácil aplicação, com o intuito de fornecer uma medida geral do grau de sonolência diurna. Na aplicação da ESE pede-se à pessoa para estimar a tendência para adormecer em oito situações monótonas da vida diária, ressaltando que não se trata de fadiga simplesmente. O entrevistado deve fornecer uma nota de zero a três, quantificando sua tendência (probabilidade) para adormecer; as notas dadas a cada uma das oito questões são somadas; o total de 9 ou mais pontos é indicativo da presença de SDE e 16 ou mais de distúrbio respiratório ou síndrome da apnéia do sono.

Está pesquisa foi aprovada pelo Comitê de Ética em pesquisa da UCDB. Os entrevistadores, devidamente treinados, receberam autorização escrita da direção da escola e do Comitê de Ética em Pesquisa para entrevistar os professores e alunos que assinaram o Termo de Consentimento Livre e Esclarecido.

$\mathrm{Na}$ análise estatística foram usados os testes qui-quadrado e o teste de análise de variância, com um nível de significância de 5\%.

\section{RESULTADOS}

A maioria dos estudantes era do sexo masculino (50,3\%), porquanto 39,2\% ingeriram bebida alcoólica e 6,6\% tabaco, 33\% já tinham apresentado depressão e 55,8\% SDE (Tabela 1).

A tabela 2 apresenta a distribuição da idade dos alunos em relação à ESE.

Tabela 1. Perfil dos pré-vestibulandos amostrados

\begin{tabular}{llcc}
\hline Variável & & Quantidade & Percentual \\
\hline Gênero & Feminino & 188 & 49,7 \\
& Masculino & 190 & 50,3 \\
Ingestãoálcool & Não & 228 & 60,8 \\
& Sim & 147 & 39,2 \\
Tabagismo & Não & 351 & 93,4 \\
& Sim & 25 & 6,6 \\
Depressivo & Não & 248 & 67,0 \\
& Sim & 122 & 33,0 \\
Epworth & Normal & 147 & 38,9 \\
& Sonolência excessiva & 211 & 55,8 \\
& Distúrbio respiratório & 20 & 5,3 \\
& ou apnéia do sono & & \\
\hline
\end{tabular}

Tabela 2. Média de idade em relação à escala de Epworth

\begin{tabular}{lcccc}
\hline Variável & Quantidade & $\begin{array}{c}\text { Média } \\
\text { de idade }\end{array}$ & Desvio-padrão & Há associação? \\
\hline Normal & 147 & 16,5 & 0,9 & \\
$\begin{array}{l}\text { Sonolência } \\
\text { excessiva }\end{array}$ & 210 & 16,5 & 0,6 & Não, \\
$\begin{array}{l}\text { Distúrbio } \\
\text { respiratório ou } \\
\text { apnéia do sono }\end{array}$ & 20 & 16,8 & 0,7 & p-valor $=0,107^{*}$ \\
\hline
\end{tabular}

* Foi aplicado o teste de análise de variância, com valor da estatística $\mathrm{F}=2,25$, e com 2; 374 graus de liberdade, totalizando 376 graus de liberdade.

O teste de análise de variância não demonstrou associação entre a idade dos pré-vestibulandos e a ESE. O teste do qui-quadrado não constatou associação significativa entre a 
ESE e o sexo da população amostrada $(p=0,148)$. O teste foi realizado com $95 \%$ de confiabilidade, com valor da estatística de 3,815 e 2 graus de liberdade. Foi realizado o teste de análise de variância, que não constatou associação entre a renda média dos pré-vestibulandos e a ESE $(p=0,454)$. Foi constatada diferença significativa entre a ingestão de álcool e a ESE ( $p<0,001)$, demonstrando associação entre as variáveis. 0 valor da estatística foi de 24,31 e com dois graus de liberdade. Foi realizado o teste qui-quadrado entre a ESE e às vezes em que os pré-vestibulandos ingeriam álcool, constatando que não houve diferença significativa entre as variáveis. O valor da estatística foi 2,188 e com dois graus de liberdade.

Foi constatada diferença significativa entre as variáveis tabagismo e a ESE $(p=0,046)$. O valor da estatística foi 3,998, com um grau de liberdade. Não foi constatada associação entre histórico de depressão e a ESE ( $p=0,312)$. O valor da estatística do teste foi de 2,333 com dois graus de liberdade.

\section{DISCUSSÃO}

Neste estudo, a prevalência da SDE corroborou os dados da literatura; 55,8\% tinham SDE, comparando os dados das muIheres $(60,6 \%)$ em relação aos homens $(51,1 \%)$. SaarempaaHeikkila et al. (1995 apud Joo et al., 2005) encontraram em sua pesquisa a prevalência de SDE em 20\% dos meninos e 22\% das meninas de 7 a 17 anos. Liu et al. (apud Joo et al., 2005), em pesquisas realizadas com sujeitos de idade entre 30 e 39 anos, também relataram que as mulheres apresentaram maior ocorrência de SDE comparado com os homens (18,7\% dos homens e 20,7\% das mulheres). Tagaya et al. (2004), por meio dos instrumentos de avaliação de sonolência versão japonesa, PSQI-J, Questionário de Saúde Geral de 12 itens (GHQ 12-J) e um questionário sobre características sociodemográficas, vida diária, situações domésticas e saúde física percebida, apontaram em pesquisa com estudantes de 34 escolas japonesas que a hora para deitar era retardada à medida que a série escolar aumentava e que, apesar de os homens deitarem mais tarde e acordarem mais tarde, as mulheres é que dormiam menos por causa do estilo de vida. Ohida et al. (2004) observaram também que a duração média de sono de adolescentes japoneses com 12 a 18 anos é menor que a dos adultos, e que na pesquisa com 107.907 sujeitos, a SDE foi encontrada em, aproximadamente, um terço dos meninos (33,3\%) e das meninas (39,2\%) e aumentaram à medida que a série aumentava. Estes índices foram maiores do que os encontrados no estudo sul-coreano com 3.871 estudantes adolescentes, em que se identificaram 15,9\% de SDE, sendo 14,9\% em meninos e 18,2\% em meninas (Joo et al., 2005).

Em outros estudos não foi identificada relação entre SDE e sexo, mas associação entre SDE com outras variáveis biopsicosocioculturais. Esta realidade é apontada por Danda et al. (2005), que não encontraram correlação significativa entre os sexos, mas observou que 99,02\% dos estudantes apresentaram hábitos de sono inadequados, destes 39,26\% com SDE. Pallos et al. (2005) encontraram 2,2\% de SDE em estudantes internacionais de pós-graduação e 19,8\% dos sujeitos com problemas de insônia. Em outro estudo com estudantes de graduação, Pallos et al. (2004) identificaram a prevalência de $4,1 \%$ de SDE e 3,2\% de acidentes ocorridos em razão de problemas de sono. Segundo Resta et al. (2003), a SDE também foi encontrada relacionada a obesidade e a síndrome de apnéia do sono, presente em 34,7\% dos pacientes obesos de 16 a 75 anos. No trabalho de Souza et al. (2002), foi verificada a prevalência de SDE e a presença de insônia no sexo masculino.

Relataram sinais e sintomas de depressão 33\% dos estudados, a maioria $(59,8 \%)$ apresentou índices de SDE. Apesar do resultado encontrado, não foi encontrada associação entre o histórico de depressão e a ESE. De acordo com Moreira e Domingues (2001 apud Reimão, 2001), em uma pesquisa realizada na cidade de Barueri-SP, com 45 sujeitos de ambos os sexos, idade entre 6 anos e dois meses e 86 anos e sete meses, estabeleceram uma relação entre a acupuntura e a psicoterapia como uma possível forma de tratamento e cura da insônia, ansiedade e depressão.

Kaplan et al. (1997) relatam que a prevalência de depressão na população dos Estados Unidos é de 3\% a 5\%, visto que o risco de depressão ao longo da vida é de 3\% a 12\% para os homens e 20\% a 26\% para as mulheres. Já a prevalência de transtornos afetivos em adolescentes é de aproximadamente $5 \%$ e, as mulheres são, em média, duas vezes mais acometidas que os homens, a partir da adolescência. Para Sadler (1991), a depressão está relacionada a vários problemas, como os de ordem sexual, acadêmicos, abuso de drogas, desordens de conduta, transtornos de ansiedade, déficit de atenção, pâni$\mathrm{co}$, desordens alimentares, entre outros, sendo considerada um transtorno com significativos níveis de comorbidade, ou seja, outros transtornos mentais, como a SDE.

$\mathrm{Na}$ presente pesquisa houve importantes associações entre os problemas de sono e as variáveis relacionadas ao consumo esporádico de álcool e tabaco. Foi verificado que $63,9 \%$ dos estudantes que ingerem álcool e $80 \%$ daqueles que consomem tabaco apresentam SDE. A intoxicação aguda com álcool induz ao sono, e é seguida pelos efeitos da abstinência e pela insônia, na segunda metade da noite (Souza; Guimarães, 1999). No levantamento nacional sobre consumo de drogas entre estudantes dos ensinos fundamental e médio, realizado pelo Centro Brasileiro de Informações sobre Drogas Psicotrópicas (Cebrid) na região centro-oeste, constatou-se que $65,5 \%$ dos entrevistados declararam já ter feito uso do álcool e 22,4\% uso de tabaco (Galduroz et al., 2004). Gasparini (2003), em um trabalho para verificação do uso de drogas lícitas e ilícitas entre 1.050 graduandos de uma Universidade de Campo Grande, MS, verificou a prevalência do uso de álcool seguida do tabaco. Na distribuição das freqüências simples e relativas do consu- 
mo de álcool, foi detectado $92,69 \%$ de uso na vida, 14,23\% de uso freqüente, $9,45 \%$ de uso pesado, e em relação ao tabaco, foi encontrado $42,88 \%$ de uso na vida, 3,31\% de uso freqüente e $9,55 \%$ de uso pesado.

Shin et al. (2003), em um estudo realizado na Coréia com 3.871 alunos com idade média de 16,8 anos, observaram que o tempo de sono total médio era insuficiente para adolescência durante a puberdade (6,3 horas/dia para estudantes homens e 6,5 horas/dia para estudantes mulheres). Pois os horários escolares que exigem que os adolescentes acordem mais cedo, as maiores demandas acadêmicas requerendo que fiquem acordados até tarde, as oportunidades sociais em expansão e o maior acesso a drogas e álcool, exigem uma maior disponibilidade e assim um tempo de sono insuficiente para esses adolescentes, ocasionando SDE.

De acordo com Ohida et al. (2004), ser do sexo feminino, ser um aluno sênior de escola média e ter um estilo de vida não saudável, dentre eles, fumar e ingerir álcool, possuem uma relação direta com problemas de sono. Os autores citam um estudo desenvolvido com adolescentes italianos, que afirma que o sono ruim possui relação significativa com a ingestão de álcool e está significativamente relacionado com problemas de sono. Johnson et al. (2003 apud Ohida et al., 2004), por meio de um estudo com 13.831 adolescentes americanos, afirmam também que fumar e fazer uso de álcool está significativamente relacionado com problemas de sono. Joo et al. (2005) constataram em uma análise com estudantes de escola média na Coréia, que os sujeitos com SDE possuíam muito mais chance de fumar cigarros ou beber álcool do que aqueles sem SDE. Os autores ainda associaram a sonolência diurna excessiva à queda no desempenho escolar.

\section{CONCLUSÕES}

Foi alta a prevalência de SDE, sendo detectadas associações entre as variáveis uso esporádico de álcool e fumo, em relação à ESE.

\section{REFERÊNCIAS}

Akerstedt TG, Fredlund P, Jansson B, Gillberg M. A prospective study of fatal accidents and sleep disturbances, fatigue, work stress, and work hours. Sleep, 24: A123, 2001.

Benbadis SR. Daytime sleepiness: when is it normal? When to refer? Cleve

Clin J Med, 65(10): 543-9, 1998.

Beusterien KM, Rogers AE, Walsleben JA, Emsellem HA, Reblando JA, Wang L, Goswami M, Steinwald $B$. Health-related quality of life effects of modafinil for treatment of narcolepsy. Sleep, 22(6): 757-65, 1999

Bulgari ML, Freitas KL. Aprendizagem e sono: relações possíveis. In: Reimão, R. Avanços em medicina do sono. São Paulo: Zeppelini Editorial, 2001

Canani SF, Barreto SSM. Sonolência e acidentes automobilísticos. J Pneumol, 27(2): 94-6, 2001.

Connor J, Norton R, Ameratunga S, Robinson E, Wigmore B, Jackson R. Prevalence of driver sleepiness in a random population-baSDE sample of car driving. Sleep, 24(6): 688-94, 2001.

Danda GJN, Ferreira GR, Azenha M, Souza KFR, Bastos 0. Sleep-wake cycle pattern and excessive daytime sleepiness in medical students. J Bras Psiquiatr 54(2): 102-106, 2005.
Drummer OH, Gerostamoulos J, Batziris H, Chu M, Caplehorn JR, Robertson MD, Swann P. The incidence of drugs in drivers killed in Australian road traffic crashes. Forensic Sci Int, 134(2-3): 154-62, 2003.

Ferreira CEC. Acidentes com motoristas no transporte rodoviário de produtos perigosos. São Paulo Perspec, 17(2): 68-80, 2003.

Franco RA, Rosenfeld RM, Rao M. First place-resident clinical science award 1999. Quality of life for children with obstructive sleep apnea. Otolaryngol Head Neck Surg. 2000;123(1 Pt 1):9-16.

Galduroz JCF, Noto AR, Fonseca AM, Carlini EA. V Levantamento nacional sobre o consumo de drogas psicotrópicas entre estudantes do ensino fundamental e médio da rede pública de ensino nas 27 capitais brasileiras. São Paulo: Cebrid/Unifesp; 2004

Gasparini HD. Uso de drogas entre estudantes universitários [dissertação de Mestrado]. Campo Grande: Universidade Católica Dom Bosco. Campo Grande; 2003.

Glina DMR, Rocha LE, Batista ML, Mendonça MGV. Saúde mental e trabalho: uma reflexão sobre o nexo com 0 trabalho e 0 diagnóstico, com base na prática. Cad Saúde Pública, 17(3): 607-16, 2001.

Guilleminault C, Stoohs R, Clerk A. Daytime somnolence: therapeutic approaches. Neurophysiol Clin, 23(1): 23-33, 1993

Häkkänen H, Summala H, Partinen M, Tiihonen M, Silvo J. Blink duration as an indicator of driver sleepiness in professional bus drivers. Sleep, 22(6): 798-802, 1999.

Häkkänen J, Summala H. Sleepiness at work among commercial truck drivers. Sleep, 23(1): 49-57, 2000

Johns MW. A new method for measuring daytime sleepiness: the Epworth sleepiness scale. Sleep, 14(6): 540-5, 1991

Joo S, Shin C, Kim J, Yi H, Ahn Y, Park M, Kim J, Lee S. Prevalence and correlates of excessive daytime sleepiness in high school students in Korea. Psychiatry and Clinical Neurosciences, 59(4): 433-440, 2005

Kaplan HI, Sadock BJ, Grebb JA. Compêndio de psiquiatria: ciências do comportamento e psiquiatria clínica. 7a ed. Trad. Dayse Batista. Porto Alegre: Artes Médicas, 1997.

Krieger J. Clinical approach to excessive daytime sleepiness. Sleep, 23(Suppl4): S95-8, 2000

McArdle N, Kingshott R, Engleman HM, Mackay TW, Douglas NJ. Partners of patients with sleep apnoea/hypopnoea syndrome: effect of CPAP treatment on sleep quality and quality of life. Thorax, 56(7): 513-8, 2001

McCartt AT, Rohrbaugh JW, Hammer MC, Fuller SZ. Factors associated with falling asleep at the wheel among long-distance truck drivers. Accid Anal Prev, 32(4): 493-504, 2000

Melamed S, Oksenberg A. Excessive daytime sleepiness and risk of occupational injuries in nonshift daytime workers. Sleep, 25(3): 315-22, 2002.

Ohida T, Osaki Y, Doi Y, Tanihata T, Minowa M, Suzuki K, Wada K, Suzuki K, Kaneita Y. An epidemiologic study of self-reported sleep problems among japanese adolescents. Sleep, 27(5): 978-85, 2004.

Pallos H, Gergely V, Yamada N, Miyazaki S, Okawa M. Exploring the quality of sleep on long-term sojourn: International graduate students in Japan. Sleep and Biological Rhythms, 3(3): $142-148,2005$

Pallos H, Yamada N, Doi Y, Okawa M. Sleep habits, prevalence and burden of sleep disturbances among japanese graduate students. Sleep and Biological Rhythms, 2(1): 37-42, 2004.

Reimão R. Avanços em medicina do sono. São Paulo: Associação Paulista de Medicina, 2001.

Resta 0, Foshino B, Bonfitto P, Giliberti T, Depalo A, Pannacciulli N, De Pergola G. Low sleep quality and daytime sleepiness in obese patients without obstrutive sleep apnoea syndrome. Journal of Internal Medicine, 253: 536-543, 2003.

Sadler LS. Depression in adolescents. Context, manifestations and clinical management. Nurses Clinical North American, 26 (3): 559-572, 1991

Saletu B, Anderer P, Saletu-Zyhlarz GM. Nichtorganische hypersomnie - epidemiologie, diagnose und therapie. Wien Klin Wochenschr, 113/7-8: 266-77, 2001.

Shin CKJ, Lee S, Ahn Y, Joo S. Sleep habits excessive daytime sleepiness and school performance in high school students. Psychiatry Clin Neurosci, 57(4): 451-453, 2003.

Souza JC. 0 sono das crianças escolares. Campo Grande: Sólivros, 1999.

Souza JC, Guimarães LAM. Insônia e qualidade de vida. Campo Grande: UCDB, 1999.

Souza JC, Magna LA, Reimão R. Excessive daytime sleepiness in Campo Grande general population, Brazil. Arq Neuropsiquiatr, 60(3-A): 558-62, 2002.

Tagaya H, Uchiyama M, Ohida T, Kamei Y, Shibui K, Ozaki A, Tan X, Suzuki H, Aritake S, Li L, Takahashi K. Sleep habits and factors associated with short sleep duration among Japanese high-school students: a community study. Sleep and Biological Rhythms, 2: 57-64, 2004.

Yang EH, Hla KM, McHorney CA, Havighurst T, Badr MS, Weber S. Sleep apnea and quality of life. Sleep, 23(4): 535-41, 2000 